\title{
Inguinal Bladder Hernia: Case Report ${ }^{*}$
}

\author{
Guo Liang Yong ${ }^{1}$, Mun Yee Siaw ${ }^{2}$, Amelia Jia Ling Yeoh ${ }^{3}$, George Eng Geap Lee ${ }^{2 \#}$ \\ ${ }^{1}$ University of Aberdeen, Aberdeen, UK \\ ${ }^{2}$ Monash University Sunway Campus, Bandar Sunway, Malaysia \\ ${ }^{3}$ University of Adelaide, Adelaide, Australia \\ Email: \#georgeeglee@msn.com
}

Received June 17, 2013; revised July 16, 2013; accepted July 24, 2013

Copyright (c) 2013 Guo Liang Yong et al. This is an open access article distributed under the Creative Commons Attribution License, which permits unrestricted use, distribution, and reproduction in any medium, provided the original work is properly cited.

\begin{abstract}
Sliding inguinal scrotal herniation of the bladder is rare but well-documented in the medical literature. The majority of the patients are asymptomatic and the hernia is usually diagnosed incidentally. Most authors had advocated surgically repair of the hernia with the reduction or resection of the bladder as the definitive management. We present an unusual case of inguinal scrotal herniation of the bladder presented as acute abdominal pain and treated with conservatively with urethral catheterization.
\end{abstract}

Keywords: Inguinal Bladder Hernia; Conservative Management

\section{Introduction}

Inguinal hernia is one of the most common surgical presentations, and approximately 20 million surgical repairs are performed annually worldwide [1]. Involvement of the urinary bladder as a sliding inguinal scrotal hernia is rare. The majority of patients with such condition are asymptomatic and the urinary bladder herniation is usually diagnosed intra-operatively [2]. The surgical reduction or resection of the bladder is usually carried out during the hernia repair as the definitive treatment for the sliding hernia. We present a rare case of a sliding inguinal bladder hernia presented as acute abdominal pain mimicking renal colic. After the Computed Tomography Urogram (CTU) demonstrating the sliding urinary bladder inguinal herniation, the patient was successfully treated with conservative management of catheterization.

\section{Case Report}

A previously well 79-year-old man presented to the Accident and Emergency Department with sudden onset of right iliac fossa pain consistent with renal colic. The patient also complained of voiding and storage lower urinary tract symptoms three days prior to the admission. Examination revealed a palpable urinary bladder and a non-tender mass arising from the right inguinal region

\footnotetext{
*Competing Interests: The authors declare that they have no competing interest.

\#Corresponding author.
}

extending into the scrotum, consistent with non-reducible ilio-inguinal hernia. The patient was able to urinate and the urine microscopy demonstrated microscopic heamaturia. In view of the symptoms of renal colic, a CTU was conducted which demonstrated a right inguinal hernia with the sliding contents of the right-sided wall of the urinary bladder and omental fat (Figure 1). The bladder demonstrated post void residual and no bowel loops were visible in the hernia.

The patient was treated with urinary bladder catheterization which drained $300 \mathrm{~mL}$ of clear urine. Upon catheterization, the ilio-inguinal hernia reduced and the patient experienced instant pain relieved. After 24 hours of oral Tamsulosin (400 mcg OD), the patient had a successful trial of void and became symptom-free. One year following the initial presentation, he attended the follow-up with no further recurrence of the hernia

\section{Discussion}

Herniation of the urinary bladder accounts for $1 \%$ to $3 \%$ of all inguinal hernias [3]. It is frequently unilateral, on the right side with a $70 \%$ male predominance [4]. Other risk factors include older age, obesity and history of herniorrhaphy [5]. Two main factors in development of bladder hernia are the presence of lower urinary tract obstruction which leads to bladder distension together with weakening of both the abdominal and the bladder wall which enables it to slide through the dilated inguinal ring, especially with constant increase in the pelvic 


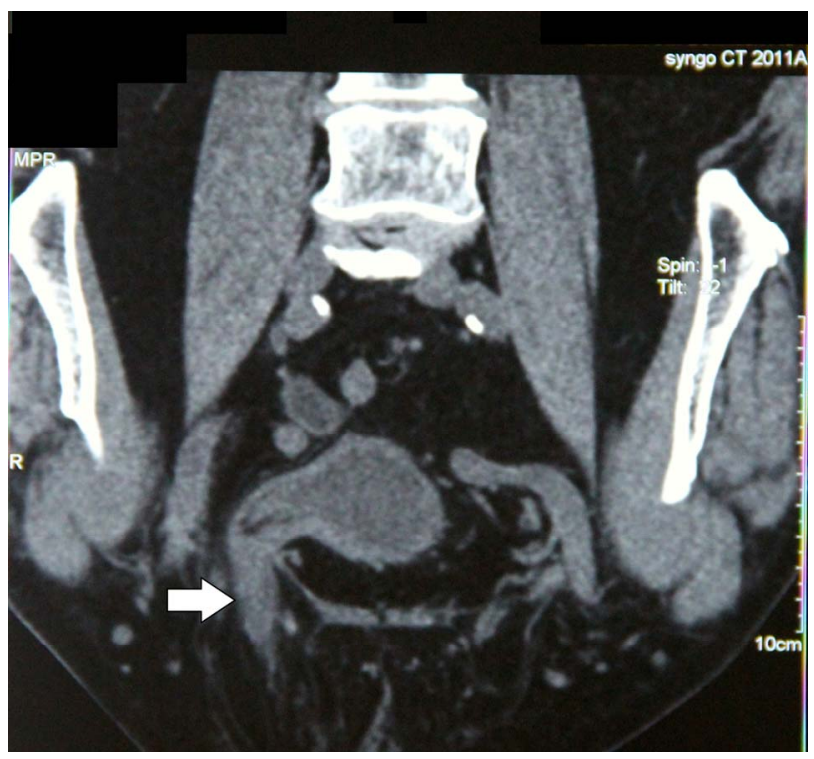

Figure 1. Right inguinal hernia, right-sided wall of the urinary bladder and omental fat.

pressure during straining.

Urinary bladder hernias are often asymptomatic and diagnosed incidentally during surgical intervention intraoperatively [6]. The acute presentation of such sliding hernia is rare, especially mimicking the symptoms of renal colic.

Radiologic investigation such as CTU is a common investigative modality for urolithiasis. Such investigation has the added advantage of identifying other or concomitant pathology that may cause abdominal pain. The diagnostic features for sliding inguinal scrotal hernia involving urinary bladder are distinctive and clear cut on CTU even without the utilization of intra-venous contrast.

The treatment of sliding urinary bladder inguinalscrotal hernia depends on the size of the hernia and the extent of the bladder involvement. Most authors in the literature had advocated surgical repair of inguinal hernia with bladder wall resection, especially with strangulation or necrosis of the bladder wall $[7,8]$. In our experience, the simple urinary bladder catheterization has the effect of de-compressing the bladder distension which may result in the reduction of the sliding hernia. The maneuver also has the effect of relieving concomitant urinary retention and pain relief. The efficacy also seems to be sustained one year following the treatment. In our best knowledge, this is the first case report of a sliding inguinal scrotal hernia involving urinary bladder presented with acute abdominal pain that resolved with catheterization.

\section{REFERENCES}

[1] J. B. Mabula and P. L. Chalya, "Surgical Management of Inguinal Hernias at Bugando Medical Centre in Northwestern Tanzania: Our Experiences in a Resource-Limited Setting,” BMC Research Notes, Vol. 5, No. 2012, pp. 585-586. doi:10.1186/1756-0500-5-585

[2] K. H. Kraft, S. Sweeney, A. S. Fink, C. W. Ritenour and M. M. Issa, "Inguinoscrotal Bladder Hernias: Report of a Series and Review of the Literature," Canadian Urological Association Journal, Vol. 2, No. 6, 2008, pp. 619-623.

[3] N. Andac, N. Baltacioglu, D. Tuney, et al., "Inguinoscrotal Bladder Herniation: Is CT a Useful Tool in Diagnosis?” Clinical Imaging, Vol. 26, No. 5, 2002, pp. 347-348. doi:10.1016/S0899-7071(02)00447-3

[4] A. A. Wagner, P. Arcand and M. H. Bamberger, "Acute Renal Failure Resulting from Huge Inguinal Bladder Hernia,” Urology, Vol. 64, No. 1, 2004, pp. 156-157. doi:10.1016/j.urology.2004.03.040

[5] A. R. Koontz, "Sliding Hernia of Diverticula of Bladder," Archives of Surgery, Vol. 70, No. 3, 1955, pp. 436-438. doi:10.1001/archsurg.1955.01270090114025

[6] M. T. Oruc, A. Z. Akbulut, O. Ozozan, et al., "Urological Findings in Inguinal Hernias: A Case Report and Review of the Literature,” Hernia, Vol. 8, No. 1, 2004, pp. 76-79. doi:10.1007/s10029-003-0157-6

[7] J. D. Casas, A. Mariscal and E. Barluenga, "Scrotal Cystocele: US and CT Findings in Two Cases," Computerized Medical Imaging and Graphics, Vol. 22, No. 1, 1998, pp. 53-56. doi:10.1016/S0895-6111(98)00007-X

[8] B. B. Carolina, G. N. Ricardo, A. C. Luis, et al., "Bladder Hernia,” Archivos Españoles de Urología, 2011, Vol. 64, No. 5, pp. 465-467. 